\title{
Pressure Dressings Versus Nonpressure Dressings After Hemorrhoidectomy: Study Protocol for a Randomized Controlled Trial
}

\section{Ping Xue}

West China School of Medicine: Sichuan University West China Hospital https://orcid.org/0000-00024587-4954

Jing Wu

Sichuan University West China Hospital

Ping Zhu

Sichuan University West China Hospital

\section{Dan Wang}

Sichuan University West China Hospital

Mei Xu

Sichuan University West China Hospital

Yi Zhang

Sichuan University West China Hospital

Guanmao Lu

Sichuan University West China Hospital

\section{Quanyi Chen}

Sichuan University West China Hospital

\section{Qin Zhang}

Sichuan University West China Hospital

\section{Renjing Tang}

Sichuan University West China Hospital

Jinbo Fang (D1145619397@qq.com )

West China School of Medicine: Sichuan University West China Hospital

\section{Study protocol}

Keywords: Hemorrhoids, Hemorrhoidectomy, Urinary retention, Postoperative pain, Postoperative bleeding, Pressure dressing, Nonpressure dressing

Posted Date: June 28th, 2021

DOI: https://doi.org/10.21203/rs.3.rs-487275/v1 
License: (c) (i) This work is licensed under a Creative Commons Attribution 4.0 International License. Read Full License

Version of Record: A version of this preprint was published at Trials on November 13th, 2021. See the published version at https://doi.org/10.1186/s13063-021-05750-3. 


\section{Abstract}

\section{Background}

Pressure dressings have been used after open hemorrhoidectomy to protect surgical wounds and manage postoperative bleeding for many years. However, pressure dressings may increase the incidence of postoperative complications, such as urinary retention, medical adhesive-related skin injury, and pain. A previous controlled trial included 67 patients who underwent Milligan-Morgan hemorrhoidectomy. The data indicated that the use of a nonpressure dressing after hemorrhoidectomy reduces the incidence of urinary retention and catheterization. However, the incidence of severe postoperative bleeding and other postoperative complications was not assessed. There is no consensus on whether it is necessary and beneficial to use a nonpressure dressing after hemorrhoidectomy. The results of this randomized clinical study will help answer this question.

\section{Methods}

In this study, we plan to include 186 patients who have undergone Milligan-Morgan hemorrhoidectomy. The purpose is to determine whether the use of nonpressure dressings after open hemorrhoidectomy is inferior to the use of pressure dressings in terms of severe postoperative bleeding and postoperative complications. The primary endpoints of the trial are the incidence of urinary retention within 24 hours after surgery and the incidence of severe postoperative bleeding one hour after dressing removal, which requires revision surgery within 24 hours after the surgery. The secondary endpoints of the study are the pain score, anal distension score, postoperative use of analgesics, and incidence of medical adhesiverelated skin injury, all of which will be assessed before removing the dressings. The length of hospitalization in days and hospitalization expenses will be recorded. Safety will be assessed with consideration of all adverse and severe adverse events related to the study treatment.

Discussion

The study received full ethics committee approval. The first patient was enrolled on 27 November 2020. The results of this trial will finally answer the question of whether a nonpressure dressing after open hemorrhoidectomy is necessary and beneficial.

Trial registration

Chinese Clinical Trial Registry (registration ID: ChiCTR2000040283). Registered on 28 November. 2020. $\{2\}$ http://www.chictr.org.cn/edit.aspx?pid=58894\&htm=4 \{2b\}

\section{Introduction}

\section{Background and rationale \{6a\}}


As people's personal and work lifestyles change, the incidence of mixed hemorrhoids in China is increasing annually ${ }^{[1]}$. According to an epidemiological study, in 2016, the incidence of anorectal diseases among urban residents in China was $51.14 \%$, and the incidence of mixed hemorrhoids was the highest among these diseases, accounting for $50.28 \%$ of all anorectal diseases ${ }^{[2]}$. In the United States of America, the incidence is approximately $4 \%$, with the prevalence being highest between 45 and 65 years

of age ${ }^{[3]}$. It is estimated that approximately $50 \%$ of the German population suffers from symptomatic hemorrhoids, and some authors believe that as many as $70 \%$ of people are affected by hemorrhoids ${ }^{[4]}$. Mixed hemorrhoids can occur at any age, and the symptoms include itching, pain, bleeding, swelling, prolapse of an anal tumor, foreign body sensation, etc. ${ }^{[5]}$. Although mixed hemorrhoids are not fatal or malignant, these symptoms can reduce a patient's quality of life and have negative psychological and physical effects ${ }^{[6]}$.

The treatment types include conservative treatment and surgical treatment. Mixed hemorrhoids with mild symptoms can be treated conservatively ${ }^{[7]}$. However, surgery is the initial treatment of choice in patients with symptomatic grade III-IV hemorrhoids ${ }^{[5,6,8]}$. Various surgical procedures have been used to treat symptomatic hemorrhoids in recent years, but (Milligan-Morgan) ligation is still considered the standard surgical procedure ${ }^{[9-13]}$. At the end of the operation, the wound is bandaged by a pressure dressing, which is the most traditional type of dressing that has been used clinically for many years ${ }^{[14]}$. The main purpose of pressure bandaging is to stop bleeding, but it may increase the incidence of postoperative complications, such as urinary retention, pain, anal swelling, and medical adhesive-related skin damage ${ }^{[12,15-18]}$. In addition, the necessity of pressure dressing after hemorrhoidectomy has received little scientific attention. A previous controlled trial included 67 patients who underwent Milligan-Morgan hemorrhoidectomy ${ }^{[19]}$. The data indicated that the use of a nonpressure dressing after hemorrhoidectomy reduces the incidence of urinary retention and catheterization. However, the incidence of severe postoperative bleeding and other postoperative complications was not assessed. There is no consensus on whether it is necessary and beneficial to use a nonpressure dressing after hemorrhoidectomy. The results of this randomized clinical study will preliminarily help answer this question.

\section{Objectives $\{7\}$}

The primary purposes of this study are to determine whether the incidence of postoperative urinary retention and pain scores of patients with nonpressure dressings after hemorrhoidectomy are lower than those of patients with pressure dressings and whether nonpressure dressings are noninferior to pressure dressings in terms of the incidence of severe postoperative bleeding requiring surgical revision.

The secondary purposes are to compare the incidence of medically adhesive skin lesions, the anal edema score, the anal dilatation score, and the rate of analgesic use between the pressure dressing group and the nonpressure dressing group. Hospitalization costs and length of stay will also be compared between the two treatment groups. 
To assess safety, the incidence of adverse events (AEs) and severe adverse events (SAEs) between the two study groups will be compared to illustrate the potential risk to patients with nonpressure dressings after hemorrhoidectomy.

\section{Trial design $\{8,9\}$}

This is a single-blind, randomized controlled study that will be conducted in the Department of Integrated Traditional Chinese and Western Medicine at West China Hospital in Sichuan University, China (Fig. 1). Eligible participants who meet the clinical criteria for mixed hemorrhoids of the Professional Committee of Colorectal Diseases, Chinese Society of Integrated Chinese and Western Medicine will be enrolled. The trial was approved by the Ethics Committee on Biomedical Research at West China Hospital of Sichuan University (approval code: NO. 2020-549). It was also registered in the Chinese Clinical Trial Registry (registration ID: ChiCTR2000040283).

A total of 186 participants with mixed hemorrhoids will be randomly assigned to two groups at a $1: 1$ ratio. After randomization, the participants in the two groups will undergo surgical treatment with Milligan-Morgan hemorrhoidectomy under general anesthesia. The incision for external hemorrhoids is fully sutured to stop bleeding. There is no active bleeding after surgery. The experimental group and control group will be treated with nonpressure dressings and pressure dressings, respectively. The timeline for the enrollment process, intervention, and follow-ups are summarized in a SPIRIT figure (Fig. 2). $\{13\}$

\section{Eligibility criteria $\{10\}$}

\section{Inclusion criteria}

The inclusion criteria are as follows:

1. Grade III-IV mixed hemorrhoids;

2. Aged $18 \sim 65$ years old;

3. Having the willingness to cooperate during the study;

4. Having provided informed consent;

5. The absence of a history of surgery for anorectal diseases. Note: Only the patients who meet the above 5 items will be included in the study.

\section{Exclusion criteria}

The exclusion criteria are as follows:

1. Patients with degree IV circular mixed hemorrhoids;

2. Women who are breastfeeding, pregnant, or menstruating; 
3. Patients with tuberculosis, diabetes, or cardiovascular, cerebrovascular, liver, kidney or hematopoietic system disorders;

4. Patients with anorectal inflammation, perianal abscess, tumor, ulcerative colitis, Crohn's disease, perianal skin disease, or related conditions;

5. Patients in whom the curative effect cannot be determined or who have incomplete data.

\section{Sample size $\{14\}$}

The results of this study will be compared with the two-sided, two-sample $t$ test $a=0.05, \beta=0.2$, and a sample size ratio of $1: 1$. In the preliminary trial, the incidence of urinary retention was the main outcome measure and was $44.7 \%$ in the nonpressure dressing group and $24.7 \%$ in the pressure dressing group. Considering an anticipated dropout rate of $10 \%$, we plan to include a total of 186 participants, with 93 participants per group.

\section{Recruitment \{15\}}

Researchers will recruit participants from the inpatient wards of Integrated Traditional Chinese and Western Medicine Departments. Patients eligible for the trial must meet all the inclusion criteria and exclusion criteria before enrollment. To recruit enough participants, all doctors in these departments will be informed of the trial and will be asked to contact the research assistant if they encounter potentially eligible patients. Potential participants will be screened, receive information on the trial, and then, be asked to sign informed consent forms. Patients who meet the selection criteria will undergo baseline assessments, and the eligible patients will be asked by medical staff to complete a general information form including their name, sex, age, medical history, etc.

\section{Randomization and blinding}

Randomization will be performed by an independent statistician with no clinical involvement in this trial by use of a random number generator program in Excel software (Kingsoft Office Software Co. Ltd., China). The random numbers will be used to create 2 groups, one for pressure dressings and the other for nonpressure dressings. Only one data administrator can access the random number table in the computer.

The participants will be enrolled by nursing staff and research assistants in the order of admission time. And they will be grouped by the data administrator using the table of random numbers. The two groups will use different types of dressings, and the operators who will place the dressings and the observers must know which group the patients are assigned to, so they cannot be blinded. However, the patients and statisticians will be blinded to the group assignments.

\section{Relevant concomitant care permitted or prohibited during the trial $\{11 \mathrm{~d}\}$}

Both groups of patients will receive conventional treatment and care during the perioperative period. These treatments will include fasting and drinking before surgery, the use of glycerine enema and fluid 
replacement, the postoperative use of analgesics, intravenous analgesia pumps and hemostatic drugs, if necessary. Pain therapy and prophylaxis will include muscle relaxants, local anesthetics, antiinflammatory drugs, opioids, nonsteroidal medications and other medications. During the postoperative period, the patient may receive intravenous or oral pain medication as needed. All previous and concomitant medications will be administered as per clinical routine and according to the patient's needs and demands. All information regarding the use of analgesic drugs will be recorded. Both groups of patients will use the same dose of hemocoagulase for preventive hemostasis. Prior to the commencement of the study, all investigators will receive standardized training on trial content, treatment strategies, evaluation, and quality control. Interventions will be carried out in accordance with good clinical practice guidelines.

\section{Intervention description $\{11$ a $\}$}

\section{Nonpressure dressing group}

In the nonpressure dressing group, gauze will be placed on the operative wounds of the patient after complete hemostasis is achieved, and then, the dressing will be placed on the gauze with foam adhesive dressing to avoid the perineum area. The purpose of the small layer of gauze is to prevent the foam adhesive dressing from affecting the wound and reduce bias.

\section{Pressure dressing group}

In the pressure dressing group, an operator will compress the wound with three layers of gauze (AM $9 \mathrm{~cm}$ $\times 11 \mathrm{~cm} \times 12 \mathrm{~cm}$ ) in a "tower" shape after achieving complete hemostasis. First, one layer of gauze will be folded twice over the surgical wound. Second, it will be covered directly with two layers of gauze and two pieces of silk tape $(45 \mathrm{~cm} \times 2.5 \mathrm{~cm}, 3 \mathrm{M}$ China Co., Ltd) in a "Y" shape to press the gauze firmly. The two pieces of silk tape will be fixed on both sides of the groin toward the middle of the back with moderate strength, avoiding the perineum during application.

\section{Outcome measures $\{12\}$}

\section{Primary outcome measures}

There will be two primary outcomes. The incidence of urinary retention within 24 hours will be one of these; typically, patients cannot urinate by themselves after using a hot compress or drug assistance and finally undergo retention catheterization. The change in mean hemostatic effect scores within 24 hours will be the other primary outcome. The effect of hemostasis will be graded using a 3-point ordinal scale: 0 $=$ the dressing was not soaked before removal, $1=$ there was no bleeding after changing the dressing due to excessive blood oozing, 2 = postoperative bleeding requiring surgical revision occurred.

\section{Secondary outcome measures}


1. Postoperative pain will be measured at rest with routine care at 6,18 , and 25 hours by using a visual analog scale (VAS) after the surgical procedure. The scale will range from 0-10 points, with higher scores indicating more severe pain ${ }^{[20]}: 0=$ no pain, $1 \sim 3=$ slight pain that can be tolerated, $4 \sim 6=$ pain that can be tolerated but affects sleep, $7 \sim 10=$ unbearable pain that affects not only appetite but also sleep.

2. The change in mean rectal tenesmus sensation scores within 25 hours will be graded using a 3-point ordinal scale: $0=$ no abnormal sensation; $1=$ mild anal discomfort, occasionally abnormal sensation; 2 = strong foreign-body sensation; 3 = strong foreign body sensation that is not easily relieved after rest or treatment.

3. The incidence of medical adhesive-related skin injuries will be measured. The skin condition will be assessed by a senior wound specialist nurse. If there is no skin damage, "no" will be recorded. When any of the following situations occur, "yes" will be recorded: (1) exfoliation, (2) tension blisters, (3) skin lacerations, (4) contact dermatitis, and (5) allergic dermatitis. In the statistical analysis, the incidence of medical adhesive-related skin damage will be calculated by a statistician.

4. The change in the mean anal edema score when the dressing is removed 24 hours after surgery will be graded using a 4-point ordinal scale: $0=$ no anal edema, $1=$ mild edema $(<1 / 4$ anal area $), 2=$ moderate edema ( $1 / 4$ to $1 / 2$ anal area $)$, and $3=$ severe edema $(>1 / 2$ anal area $)$.

5 . The total dose of analgesics before removing the dressing will be recorded.

6. The length of hospitalization will be recorded in days.

7. Hospitalization expenses will be recorded.

\section{Study organization}

\section{Data collection and management $\{19\}$}

The researchers will independently record demographic and clinical data for all patients. Preoperative data will be collected within 1 day after recruitment. Postoperative data will be collected within 2 days after surgery. The organizational structure of the trial is as follows. The steering committee will have full oversight over the design of the trial. The investigators of the Data Management Safety Committee (DMSC) will supervise and confirm that the case report form (CRF) is correctly completed and that the data are consistent with the original data. If there are any errors or omissions, the investigator will immediately correct them. We do not intend to collect personal information about potential or registered participants other than that typically collected during hospitalization. For confidentiality, the electronic health information will be encrypted in accordance with the hospital protocol. After the trial, personally identifiable information will be omitted and placed in a separate database for data analysis.

\section{Statistical analysis}

The validity analysis of the study will be mainly based on intention-to-treat (ITT, all randomized cases) and per-protocol analyses (PP, cases that comply with the trial protocol, good compliance, and completed 
CRF). First, the Shapiro-Wilk test will be used to test whether the quantitative data follow a normal distribution. If the data are normally distributed, the mean value and standard deviation will be used to describe the quantitative data, and a $t$ test will be used for analysis. Nonnormally distributed data will be expressed as the median and quaternary and compared using the Mann-Whitney $\mathrm{U}$ test. The qualitative data will be expressed as the frequency and percentage; non-grade data will be compared by the chisquare test, and grade data will be compared by the Mann-Whitney U test. Randomization will be performed 24 hours prior to the surgical intervention. Data will not be collected and analyzed for patients not undergoing surgical intervention. If the scheduled surgery is not performed, the study participant will be excluded from the clinical trial. For every patient who is excluded from the trial after randomization and before the start of the observation period (postoperative wound with or without compression), another study participant will be included to achieve the number of cases needed. The randomization sequence will continue. The next patient will not necessarily be assigned to the same group as the excluded patient. This research will continue until the required number of evaluable patients is reached. Due to complications, a very small number of patients may not be able to comply with the randomly assigned treatment. The study will not exclude these patients. All statistical tests will be two-sided, and $P<0.05$ will be considered statistically significant. If baseline factors are inconsistent between the two groups, subgroup analysis and multivariate analysis will be considered.

In the safety analysis, Fisher's exact test will be used to compare the incidence of AEs and SAEs by category (severity) between the two groups.

\section{Adverse events (AEs) \{22\}}

In this study, AEs are defined as any treatment-related medical events, including any treatment-related adverse and unexpected signs, symptoms or diseases. The AEs known to follow nonpressure dressing and pressure dressing treatment include severe postoperative hemorrhage. At each visit, the participants will report AEs and be examined by a physician. AEs will be evaluated and reported to the principal investigator according to Common Terminology Criteria for Adverse Events (v4.03) ${ }^{[21]}$.

\section{Auditing \{23\}}

The ethics committee will conduct local monitoring of trial quality after the first patients have been enrolled. The Trial Management Team will meet every 3 months to check the implementation of the study,including the recruitment rate, data quality, and adverse event reporting.

\section{Plans for communicating important protocol amendments to relevant parties $\{25\}$}

The protocol, statistical analysis plan, data safety management plan, informed consent forms, and recruitment materials have been reviewed and approved by the Ethics Committee on Biomedical Research at West China Hospital of Sichuan University. Any subsequent modifications will be submitted for review, and annual safety and progress reports will be presented. In addition, online trial registries will be updated accordingly. The data will be accessible through the research center upon reasonable request. 


\section{Dissemination plans $\{31$ a $\}$}

The outcomes will be disseminated through peer-reviewed publications, a master's thesis, community groups, or conference presentations. A summary of findings will be prepared for publication via social media platforms including wechat and Weibo.

\section{Discussion}

Pressure dressings are commonly used and have been considered a clinical standard for wounds after mixed hemorrhoid operations in China. However, these dressings can lead to some postoperative complications, and there are few reports on improvements in wound dressing methods after hemorrhoid surgery. This study will be carried out to determine whether nonpressure bandaging can be used routinely after open hemorrhoidectomy and whether it may be considered in specific circumstances.

During the operation, the patients will be treated with general anesthesia, and the method of applying a pressure dressing is complicated, so at least two medical staff will perform wound bandaging; nonpressure dressings need only a self-adhesive foam dressing, and only one medical staff member will be needed to perform wound dressing, and the process takes less time. The nonpressure dressing method can not only reduce the workload of medical staff but also save time.

Methods to minimize bias were implemented whenever possible. Randomization was performed 24 hours before surgery. Randomization ensures a statistically consistent baseline between the experimental and control groups. However, one limitation of this study is that the researchers and outcome assessors cannot be blinded to the group assignments. The blinding of outcome assessors is an important way to prevent bias, but unfortunately, this step cannot be carried out in this study. However, the patients can be blinded. Before conducting any trial-related procedures, the researcher will obtain a written informed consent from each potential research participant. Randomization will be performed with a random number table generated in Excel, and then, the patients will be assigned a group according to the order of admission.

The research plan is consistent with clinical practice to the greatest extent possible to ensure complete and trouble-free data collection. Observation indicators such as pain and anal swelling may have affect the evaluation at certain time points while the patient is sleeping. Missing data are expected in these cases, but they should be minimized. The research leader will bear full legal responsibility for the entire study and will report to the Department of Clinical Research Management of West China Hospital of Sichuan University.

\section{Trial status}

Participant recruitment is still being undertaken. Enrollment started in November 2020, and the trial is expected to be completed by September 2021. 


\section{Abbreviations}

AE: adverse event; Cl: confidence interval; CRF: case report forms; ITT: intention to treat; NRS: numerical rating scale; $\mathrm{D}=$ discharge; PP: per protocol; RCT: randomized controlled trial; SAE: severe adverse event; SD: standard deviation

\section{Declarations}

\section{Acknowledgments}

We thank the nursing staff members who helped collect the trial data.

\section{Funding $\{4\}$}

This work was supported by the Key Research and Development Project of Science and Technology Department of Sichuan Province, China (2020YFS0269) (to Dr. Jing Wu).

\section{Authors' contributions}

JW obtained funding for the project and conceived the study. PX designed the study and drafted the manuscript. JBF helped to design the study. DW, MX, YZ, GML, QZ ,QYC and RGT, will perform this study. $\mathrm{PZ}$ will perform the statistical analysis. The authors read and approved the final manuscript.

\section{Authors' information}

${ }^{1}$ West China School of Nursing, Sichuan University, Chengd, Sichuan, China.

${ }^{2}$ Department of Integrated Chinese and Western Medicine, West China Hospital, Sichuan University, Chengd, Sichuan, China.

${ }^{3}$ Cheng Du Shang Jin Nan Fu Hospital, West China Hospital, Sichuan University, Chengd, Sichuan, China.

Ethics approval and consent to participate $\{20\}$

The Ethics Committee on Biomedical Research, West China Hospital of Sichuan University approved the study protocol (approval code: NO. 2020-549). It was also registered in the Chinese Clinical Trial Registry (registration ID: ChiCTR2000040283). Before randomization, all participants will be requested to provide written informed consent.

\section{Competing interests $\{28\}$}

The authors declare that they have no competing interests.

\section{References}


[1] Chinese guideline for diagnosis and treatment of hemorrhoids (2020). Colorectal and anal surgery. 2020. 26(05): 519-533.

[2] Jiang Wei, Zhang Hongxi, Sui Nan, Li Rong, Yan changkai. Epidemiological survey of common anorectal diseases among urban residents in China. China public health. 2016. 32(10): 1293-1296.

[3] Mott T, Latimer K, Edwards C. Hemorrhoids: Diagnosis and Treatment Options. Am Fam Physician. 2018. 97(3): 172-179.

[4] Joos AK, Herold A. Haemorrhoidal disease. New conservative and operative treatments for a wide spread disease. Coloproctology. 2011. 33(2): 86-96.

[5] Castinel, A., Pigot, et al. Guidelines for the treatment of hemorrhoids. J Visc Surg. 2016.

[6] Muldoon R. Review of American Society of Colon and Rectal Surgeons Clinical Practice Guidelines for the Management of Hemorrhoids. JAMA Surg. 2020.

[7] Ganz RA. The evaluation and treatment of hemorrhoids: a guide for the gastroenterologist. Clin Gastroenterol Hepatol. 2013. 11(6): 593-603.

[8] Rubbini M, Ascanelli S. Classification and guidelines of hemorrhoidal disease: Present and future. World J Gastrointest Surg. 2019. 11(3): 4-8.

[9] Saylam B, Ozer MV, Duzgun AP, Dinc T, Gulseren MO, Coskun F. The milligan-morgan operation in the surgical treatment of hemorrhoids: Survey of 828 cases. Turk J Surg. 2011. 27(1): 31.

[10] Panarese A, Pironi D, Vendettuoli M, et al. Stapled and conventional Milligan-Morgan haemorrhoidectomy: different solutions for different targets. Int J Colorectal Dis. 2012. 27(4): 483.

[11] Lukasz D, Michal M, Radzislaw T, et al. Surgical treatment of haemorrhoidal disease - the current situation in Poland. Przegla『d Gastroenterologiczny. 2016. 11: 111-114.

[12] Langenbach MR, Seidel D. Tamponade dressings versus no tamponade after hemorrhoidectomy: study protocol for a randomized controlled trial. Trials. 2019. 20(1): 188.

[13] Altomare DF, Giuratrabocchetta S. Conservative and surgical treatment of haemorrhoids. Nat Rev Gastroenterol Hepatol. 2013. 10(9): 513-21.

[14] Bai Xueshan, Lin guole. Advances in surgical treatment of hemorrhoids and hemorrhoids. Colorectal and anal surgery. 2020.26 (03): 276-279

[15] Lin YH, Liu KW, Chen HP. Haemorrhoidectomy: prevalence and risk factors of urine retention among post recipients. J Clin Nurs. 2010. 19(19-20): 2771-6. 
[16] Qi-Ming X, Jue-Ying X, Ben-Hui C, Jing W, Ning L. Risk Factors for Postoperative Retention After Hemorrhoidectomy: A Cohort Study. Gastroenterol Nurs. 2015. 38(6): 464-8.

[17] Tegon G, Pulzato L, Passarella L, Guidolin D, Zusso M, Giusti P. Randomized placebo-controlled trial on local applications of opioids after hemorrhoidectomy. Tech Coloproctol. 2009. 13(3): 219-224.

[18] Patel S, Shahzad G, Rizvon K, Subramani K, Viswanathan P, Mustacchia P. Rectal ulcers and massive bleeding after hemorrhoidal band ligation while on aspirin. World Journal of Clinical Cases. 2014. 002(004): P.86-89.

[19] $\mathrm{Ni} \mathrm{Y} \mathrm{L.} \mathrm{Effect} \mathrm{of} \mathrm{different} \mathrm{dressing} \mathrm{methods} \mathrm{on} \mathrm{postoperative} \mathrm{urination} \mathrm{function} \mathrm{in} \mathrm{patients} \mathrm{with}$ mixed hemorrhoids. Jilin medical. 2014. 35(32): 7235.

[20] Hawker GA, Mian S, Kendzerska T, French M. Measures of adult pain: Visual Analog Scale for Pain (VAS Pain), Numeric Rating Scale for Pain (NRS Pain), McGill Pain Questionnaire (MPQ), Short-Form McGill Pain Questionnaire (SF-MPQ), Chronic Pain Grade Scale (CPGS), Short Form-36 Bodily Pain Scale (SF-36 BPS), and Measure of Intermittent and Constant Osteoarthritis Pain (ICOAP). Arthritis Care Res (Hoboken). 2011. 63 Suppl 11: S240-52.

[21] Cancer NIO. Common Terminology Criteria for Adverse Events (CTCAE) v4.0. 2009 .

\section{Figures}




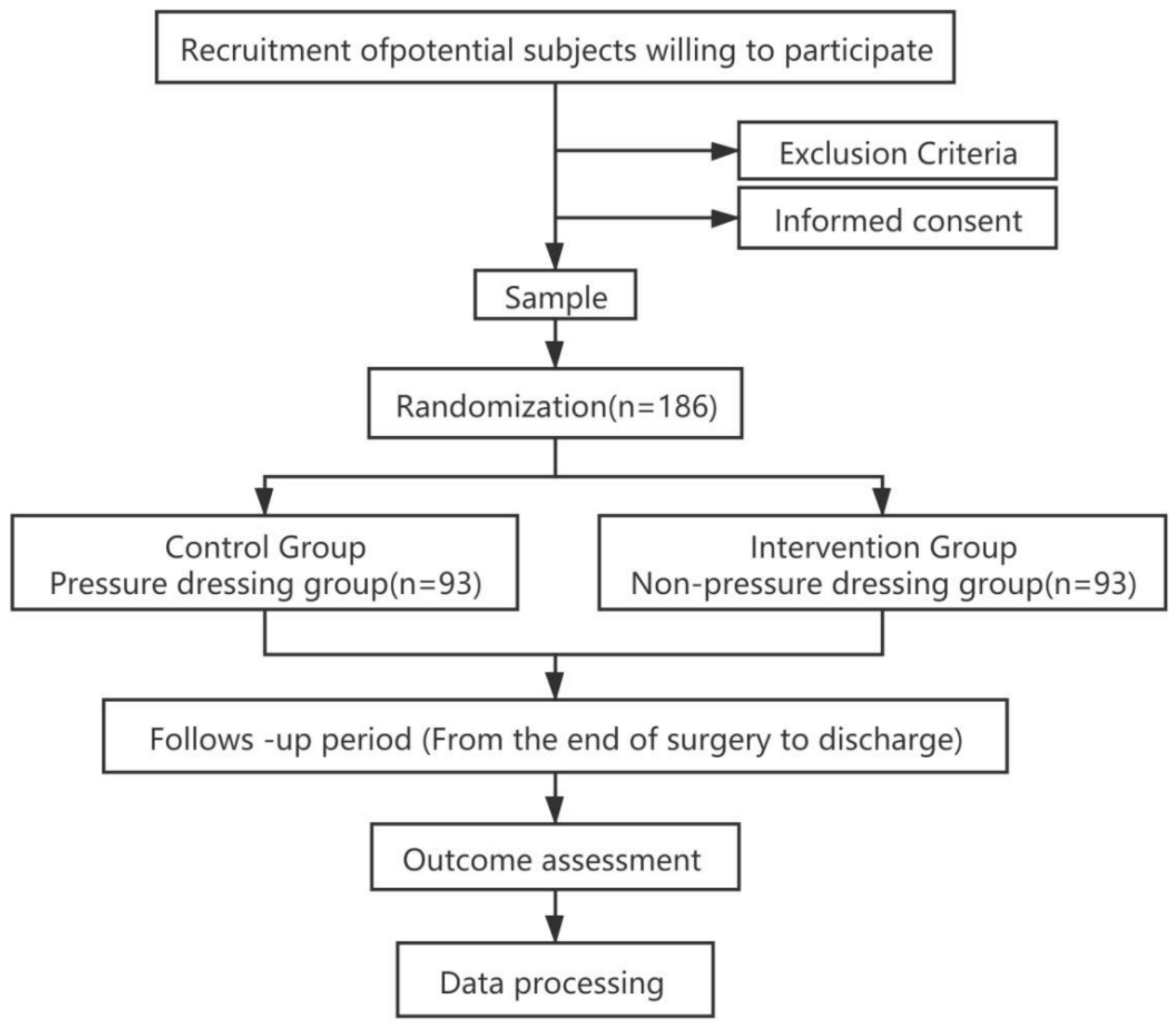

Figure 1

A flow chart of the study stages. 


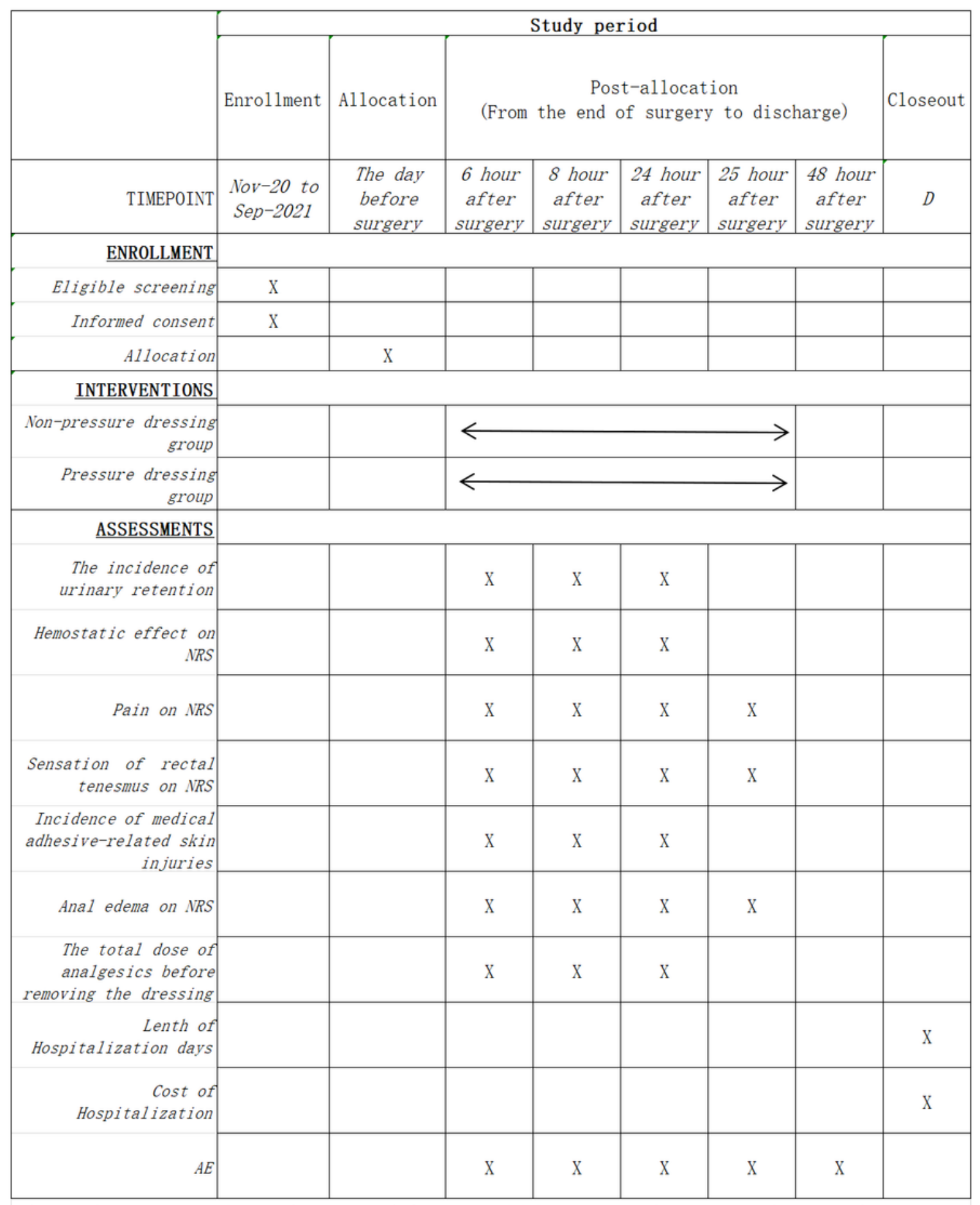

LB=Lobectomy begins, LE=Lobectomy ends, D=Discharge, AE=Adverse events aTimepoints are established based on hours post-op, not on allocation. Fig. 2 Schedule of enrolment, interventions, and assessments

\section{Figure 2}

Timeline of the enrollment process, interventions, and assessments Note: $D=D i s c h a r g e, A E=A d v e r s e$ events, NRS: Numerical rating scale

\section{Supplementary Files}


This is a list of supplementary files associated with this preprint. Click to download.

- SPIRITchecklist5.31.docx 